\title{
TaNAC29, a NAC transcription factor from wheat, enhances salt and drought tolerance in transgenic Arabidopsis
}

Quanjun Huang ${ }^{\dagger}$, Yan Wang ${ }^{\dagger}$, Bin Li, Junli Chang, Mingjie Chen, Kexiu Li, Guangxiao Yang ${ }^{*}$ and Guangyuan He*

\begin{abstract}
Background: NAC (NAM, ATAF, and CUC) transcription factors play important roles in plant biological processes, including phytohormone homeostasis, plant development, and in responses to various environmental stresses.

Methods: TaNAC29 was introduced into Arabidopsis using the Agrobacterium tumefaciens-mediated floral dipping method. TaNAC29-overexpression plants were subjected to salt and drought stresses for examining gene functions. To investigate tolerant mechanisms involved in the salt and drought responses, expression of related marker genes analyses were conducted, and related physiological indices were also measured. Expressions of genes were analyzed by quantitative real-time polymerase chain reaction (qRT-PCR).

Results: A novel NAC transcription factor gene, designated TaNAC29, was isolated from bread wheat (Triticum aestivum). Sequence alignment suggested that TaNAC29 might be located on chromosome 2BS. TaNAC29 was localized to the nucleus in wheat protoplasts, and proved to have transcriptional activation activities in yeast. TaNAC29 was expressed at a higher level in the leaves, and expression levels were much higher in senescent leaves, indicating that TaNAC29 might be involved in the senescence process. TaNAC29 transcripts were increased following treatments with salt, PEG6000, $\mathrm{H}_{2} \mathrm{O}_{2}$, and abscisic acid (ABA). To examine TaNAC29 function, transgenic Arabidopsis plants overexpressing TaNAC29 were generated. Germination and root length assays of transgenic plants demonstrated that TaNAC29 overexpression plants had enhanced tolerances to high salinity and dehydration, and exhibited an ABA-hypersensitive response. When grown in the greenhouse, TaNAC29overexpression plants showed the same tolerance response to salt and drought stresses at both the vegetative and reproductive period, and had delayed bolting and flowering in the reproductive period. Moreover, TaNAC29 overexpression plants accumulated lesser malondialdehyde (MDA), $\mathrm{H}_{2} \mathrm{O}_{2}$, while had higher superoxide dismutase (SOD) and catalase (CAT) activities under high salinity and/or dehydration stress.
\end{abstract}

Conclusions: Our results demonstrate that TaNAC29 plays important roles in the senescence process and response to salt and drought stresses. ABA signal pathway and antioxidant enzyme systems are involved in TaNAC29-mediated stress tolerance mechanisms.

Keywords: Wheat, Arabidopsis, NAC, TaNAC29, Abiotic stress, ABA-hypersensitive

\footnotetext{
*Correspondence: ygx@hust.edu.cn; hegy@hust.edu.cn

${ }^{\dagger}$ Equal contributors

The Genetic Engineering International Cooperation Base of Ministry of Science and Technology, Key Laboratory of Molecular Biophysics of Ministry of Education, College of Life Science and Technology, Huazhong University of Science \& Technology (HUST), Wuhan 430074, China
}

(C) 2015 Huang et al. Open Access This article is distributed under the terms of the Creative Commons Attribution 4.0 International License (http://creativecommons.org/licenses/by/4.0/), which permits unrestricted use, distribution, and reproduction in any medium, provided you give appropriate credit to the original author(s) and the source, provide a link to the Creative Commons license, and indicate if changes were made. The Creative Commons Public Domain Dedication waiver (http://creativecommons.org/publicdomain/zero/1.0/) applies to the data made available in this article, unless otherwise stated. 


\section{Background}

Plants are frequently challenged by unfavorable environmental conditions, including extreme temperatures, drought, and high salinity. Upon exposure to harmful environmental conditions, many related genes are induced [1]. Transcription factors (TFs) are one such related gene family. Numerous studies demonstrated that TFs play vital roles in plant gene regulation, either activating or preventing target gene expression [2, 3]. Among TFs families, NAC TFs and their corresponding cis-acting sequences act as molecular switches to regulate temporal and spatial gene expression $[2,3]$.

The NAC superfamily is one of the largest TF families in plants. Most NAC proteins share a highly conserved NAC domain at the $\mathrm{N}$-terminal, and a diversified activation domain at the C-terminal [2]. NAC TFs play a vital role in various plant developmental processes, including leaf senescence, phytohormone homeostasis, and responses to unfavorable environmental stresses [2, 3].

In Arabidopsis, overexpression of ANAC019, ANAC055, RD26/ANAC072, and ATAF1/ANAC002 confer drought tolerance [4, 5]. Plants overexpressing ATAF2/ANAC081 have a greater susceptibility to Fusarium oxysporum [6]. Overexpression of JUB1/ANAC042 and VNI2/ANAC083 delays senescence and enhances resistance to abiotic stresses [7, 8]. In rice (Oryza sativa), overexpression of OsNAC5, OsNAC9, and OsNAC10 significantly enlarges roots, and thereby enhances tolerance to drought stress, furthermore, these transgenic rice plants produce a higher grain yield under field conditions [9-11]. OsNAC6 overexpression in rice enhances tolerances to salt, drought, and low temperature stresses, but in this case the transgenic rice exhibits low grain yield and growth retardation [12]. Overexpressing OsNACO45 in rice enhances salt and drought tolerance [13]. When the rice stress-responsive NAC gene $S N A C 1$ was introduced into rice and wheat, the transgenic plants displayed significantly enhanced tolerances to multiple abiotic stresses [14, 15]. Kaneda et al. [16] revealed that overexpression of OsNAC4 leads to hypersensitive cell death, whereas, in OsNAC4 knock-down transgenic lines, hypersensitive cell death is significantly reduced. OsNAC122 and OsNAC131 proteins are involved in the response to infection by Magnaporthe grisea, and may play a role in the phytohormone-mediated signaling pathway [17].

Compared with Arabidopsis, rice, and other species, there have been fewer investigations into NAC in wheat. In bread wheat (Triticum aestivum), transgenic lines overexpressing TaNAC69 produce more biomass in the shoot and root when grown under stress-inducing conditions [18]. Overexpression of TaNAC2, TaNAC2a, and TaNAC67 in plants improves tolerances to low temperature, high salinity, and drought stresses [19-21]. Quantitative real-time polymerase chain reaction (qRT-PCR) assays suggested that
TaNAC8, TaNAC4, TtNAMB-2, and TaNAC69-1 participate in responses to various biotic and abiotic stresses [22-24]. Overall, these studies demonstrated that the factors mostly affecting expression of NAC genes are salt, drought, and extreme temperatures; and several NAC genes are simultaneously co-expressed in a developmental/organ-specific way.

In this study, a novel NAC transcription factor gene TaNAC29 was cloned from wheat. Gene expression pattern analysis demonstrated that TaNAC29 was upregulated by high salinity, dehydration, $\mathrm{ABA}$, and $\mathrm{H}_{2} \mathrm{O}_{2}$ treatments. TaNAC29 enhanced tolerance to high salinity and drought stress in transgenic Arabidopsis, and exhibited an ABAhypersensitive response. Morphological assays revealed that overexpression of TaNAC29 delayed bolting and flowering. Our results provide evidence that TaNAC29 participates in the ABA signal pathway, and plays important roles in stress responses and developmental processes.

\section{Results}

\section{TaNAC29 encodes a plant-specific NAC transcription} factor

A novel $N A C$ gene was cloned from bread wheat. This gene was designated as TaNAC29 as it had high homology to NAC29 from Aegilops tauschii. It is established that spontaneous hybridization of the wild grass Aegilops tauschii $(2 n=14$; DD) with cultivated wheat Triticum turgidum $(2 n=4 x=28 ; \mathrm{AABB})$ resulted in $T$. aestivum $(2 n=6 x=42$; AABBDD) [25]. Moreover, the Ae. tauschii genome has been sequenced, and 1489 TFs in 56 families, including the NAC family, have been identified [25]. The full length cDNA of TaNAC29 is 1198 bp long with a 1074 bp open reading frame (ORF), and encodes a protein with a predicted relative molecular mass of $38.397 \mathrm{kDa}$.

Sequence alignment and phylogenetic analysis (Additional file 1: Figures S1A and S2) revealed that TaNAC29 had $96 \%$ identity to W5BNH0 (EMBL: C116E5668.1) from T. aestivum, $92 \%$ identity to HvNAC023 (GenBank: CBZ41159.1) from Hordeum vulgare, and $89 \%$ identity to NAC29 (GenBank: EMT28859.1) from Ae. tauschii. Additionally, TaNAC29 had relative high homology with OsNAC10 (GenBank: EAZ40329.1) and ANAC047 (speedy hyponastic growth; GenBank: AEE74033.1), demonstrating their biological functions $[11,26]$. As there was high identity between TaNAC29 and W5BNH0, nucleic acid sequence alignment was conducted. This revealed that, including the ORF and untranslated region (UTR), TaNAC29 was $96.5 \%$ identical to W5BNH0 (Additional file 1: Figure S3). Comparison results indicated that TaNAC29 and W5BNH0 may be the same gene, and similar to W5BNH0, the novel TaNAC29 might be located on the 2BS chromosome. To further verify if TaNAC29 and W5BNH0 were the same gene, a wheat 
whole-genome survey was performed using the TaNAC29 sequence, and the DNA sequence with the highest identity to TaNAC29 detected. Use of a DNA splicing program revealed that, excluding the intron, this cDNA sequence was the same with the W5BNH0 sequence. Therefore, the whole-genome survey indicated that TaNAC29 gene might be the same gene with W5BNH0. To further examine whether slight differences existed, two TaNAC genes were selected from NCBI for a BlastN against EMBL; this revealed that TaNAC2D (GenBank: GQ231954.1) and TaNAC69 (GenBank: DQ022842.1) shared 96.8 and $98 \%$ identity to wheat whole-genome cDNAs, respectively. The slight difference between TaNAC29 and W5BNH0 may be a result of the different wheat cultivars used.

TaNAC29 contains a typical NAC structure with a conserved NAC domain (amino acids 15-177) including five subdomains (A: 15-35, B: 41-61, C: 70-105, D: 114-142, E: 163-177) consistent with NAC conserved domain characteristics. Four L motifs were identified at the divergent C-terminal region (amino acids 178-357) using the multiple EM for motif elicitation (MEME) tool (Additional file 1: Figure S1A and B). Transactivation activity assays confirmed that TaNAC29 was a transcriptional activator, and the C-terminal region possessed transcriptional activation activity (Additional file 1: Figure S4). Kjaersgaard et al. [27] demonstrated that the L motif is sufficient for transactivation activity of HvNAC013. Therefore, to further investigate the function of the L motif, the transactivation activity of seven truncated versions of TaNAC29 was examined. Among these, six truncated versions containing the $\mathrm{L}$ motif had transactivation activity, whereas the TaNAC29 ${ }_{1-233}$ fragment without the $\mathrm{L}$ motif had no transactivation activity (Additional file 1: Figure S4), suggesting that the $\mathrm{L}$ motif plays an important role in transactivation activity. Expression of a TaNAC29-GFP fusion protein in wheat mesophyll protoplasts demonstrated that the green fluorescent protein (GFP) and 4',6diamidino-2-phenylindole (DAPI; a nuclear stain marker) were confined to the nucleus (Additional file 1: Figure S5); this is consistent with its function as a transcription regulator. Moreover, PONDR VL3 analysis [27, 28] indicated that the C-terminal region of TaNAC29 was intrinsically disordered (ID) to a large degree (Additional file 1: Figure $\mathrm{S} 1 \mathrm{C}$ ), suggesting that the protein was largely unfolded in the C-terminal region.

\section{Expression of TaNAC29 is upregulated by abiotic stresses and signal molecules}

Temporal and spatial expression analyses revealed that TaNAC29 had relatively higher expression levels in the leaf, stem, flag leaf, and stamen, with the highest expression levels occurring in the leaf. However, TaNAC29 expressed at very low levels in the root, pistil, embryo, endosperm, coleoptile, and caryopsis (Additional file 1:
Figure S6). TaNAC29 transcripts were much higher in mature senescing leaves than in young green leaves. This suggests that TaNAC29 might be involved in the senescence process in wheat.

To investigate the response of TaNAC29 to abiotic stresses, TaNAC29 expression levels were examined following $\mathrm{NaCl}$, PEG6000, $\mathrm{H}_{2} \mathrm{O}_{2}$ and ABA treatments. qRT-PCR analysis revealed that TaNAC29 was greatly upregulated by $\mathrm{NaCl}$, PEG6000, $\mathrm{ABA}$, and $\mathrm{H}_{2} \mathrm{O}_{2}$ treatments in the leaf, and by $\mathrm{NaCl}, \mathrm{PEG} 6000$, and $\mathrm{ABA}$ treatments in the root; it was only slightly upregulated by $\mathrm{H}_{2} \mathrm{O}_{2}$ in the root (Fig. 1). Interestingly, the expression level increase in the root was stronger than in the leaf following $\mathrm{NaCl}$ and PEG treatments, suggesting a close correlation with a relative low organ-specific expression in the root. These qRT-PCR results strongly suggest that TaNAC29, like other stress-associated $N A C$ genes $[4,11,18]$, participates in plant stress responses.

\section{Salt and drought tolerances of TaNAC29-overexpression plants}

Transgenic Arabidopsis plants were generated to explore the functions of TaNAC29. Seven transgenic lines $\left(\mathrm{T}_{3}\right)$ were confirmed through kanamycin resistance analysis. Among these, three overexpression (OE) lines, designated OE1, OE2, and OE3, showed higher TaNAC29 expression levels by semi-quantitative analysis (Additional file 1: Figure S7). When grown in soil, phenotypes of the TaNAC29-overexpression plants were not significantly different from the wild type (WT) at the vegetative phase, but showed delayed bolting and flowering at the reproductive stage under normal growth conditions (Additional file 1: Figure S8). This delayed phenotype was similar to those observed in JUB1- and ATAF1-overexpressing plants [5, 7].

Next, TaNAC29-overexpression plants were examined for tolerance to salt stress. Twenty-five-day-old seedlings, grown in soil, were irrigated with $250 \mathrm{mM} \mathrm{NaCl}$ solution for 4 weeks $(4 \mathrm{w})$, WT and vector control (VC) plants had a survival rate of $\sim 20 \%$, while TaNAC29 overexpressing lines had survival rates of over $80 \%$ (Fig. 2a). When 45-day-old and 65-day-old seedlings were treated for 21 days $(21 \mathrm{~d})$ in the same way, all WT and VC plants died, whereas OE1 plants still had a survival rate of over $50 \%$ under these conditions (Fig. 2a). This indicated that overexpression of TaNAC29 could greatly enhance tolerance to salt stress.

To investigate the drought stress response of the TaNAC29-overexpression lines, twenty-five-day-old seedlings at the vegetative phase, were subjected to drought stress through withholding water for $21 \mathrm{~d}$, followed by rewatering for $7 \mathrm{~d}$. Approximately $50 \%$ of OE1 plants recovered from a dying status, whereas the survival rate 

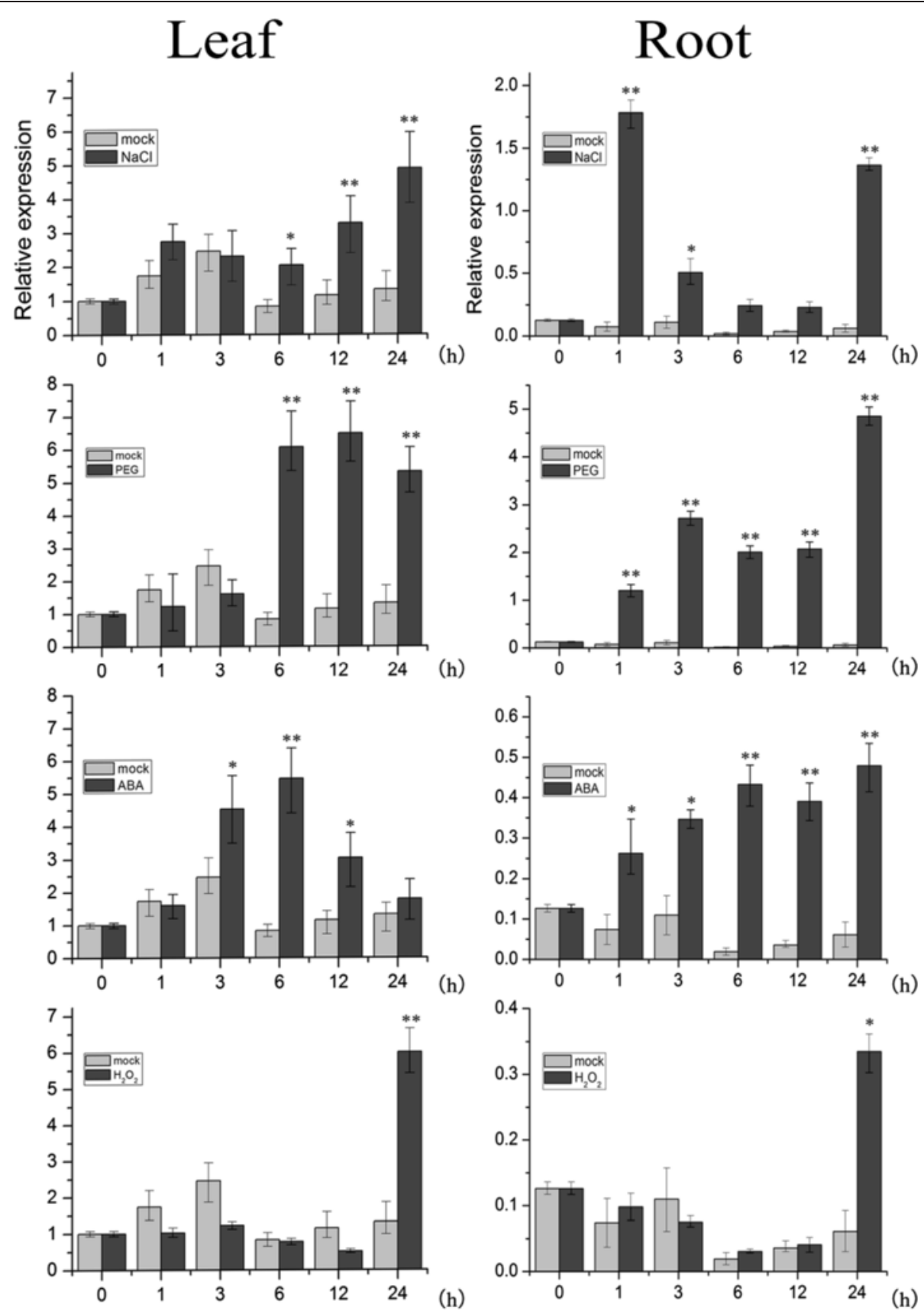

Fig. 1 Expression patterns of TaNAC29 in wheat after stress treatments. Expression patterns of TaNAC29 in wheat leaves and roots after NaCl, PEG6000, ABA and $\mathrm{H}_{2} \mathrm{O}_{2}$ treatments by qRT-PCR analysis. Leaf and root were collected after different stress treatment. The $2^{-\Delta \Delta C T}$ method was used in qRT-PCR analysis. Transcript levels were normalized to TaActin. Values are means \pm SE of three replicates. Asterisks indicate statistically significant differences from mock $\left({ }^{*} P<0.05 ;{ }^{*} P<0.01\right)$. Three independent experiments were performed

for both WT and VC plants was $15 \%$ (Fig. 2b). When 65-day-old seedlings at the reproductive stage were treated in the same way, most transgenic lines still survived, but only $20 \%$ of WT plants were recovered (Fig. 2b). These observations indicated that overexpression of TaNAC29 could confer resistance to drought stress. Finally, statistical analysis of survival rate of 25-day-old seedling after salt and drought stresses revealed that over $50 \%$ of transgenic plants were still alive, whereas $\sim 85 \%$ of WT and VC plants died (Fig. 2c).

To further verify whether TaNAC29-overexpressing plants with enhanced drought stress were associated with transpiration, the phenotype of detached leaves was examined by air-drying in a $25{ }^{\circ} \mathrm{C}$ environment. After 5 


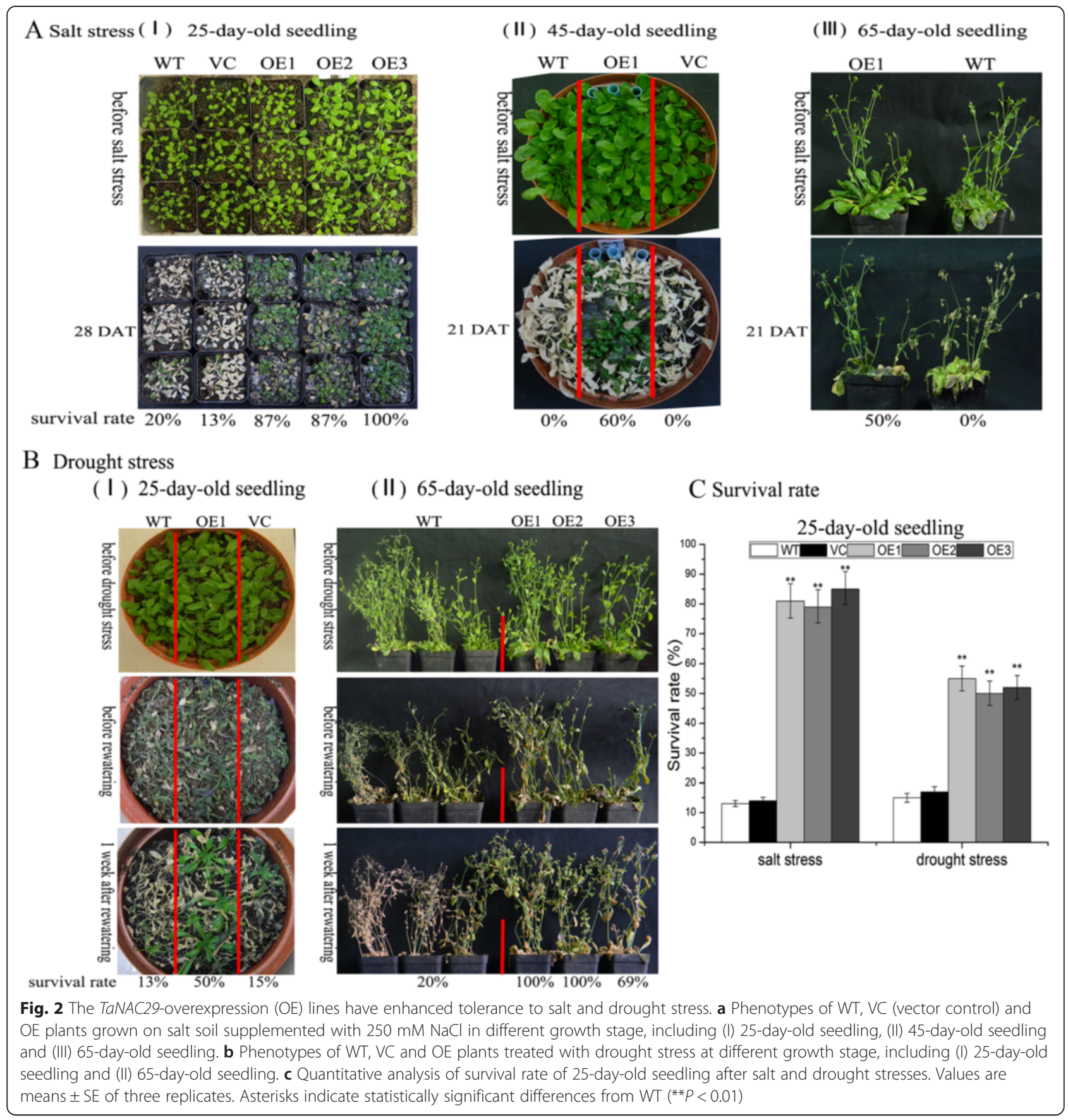

or $7 \mathrm{~h}$, the leaves of WT and VC had severely curved, whereas the transgenic plant leaves displayed a slightly curled phenotype (Additional file 1: Figure S9A). Water loss rate assays revealed that TaNAC29-overexpression lines had a lower rate of water loss at each time point (Additional file 1: Figure S9B), thus rendering TaNAC29 transgenic plants more tolerant to drought stress.

Stress tolerance of plants overexpressing TaNAC29 was further examined by root length analysis. Transgenic plants had a longer root than WT under drought stress conditions (Additional file 1: Figure S10). When grown on 1/2 Murashige-Skoog (MS) medium containing $120 \mathrm{mM} \mathrm{NaCl}$, the growth of the primary roots of TaNAC29-overexpression line seedlings was significantly stronger than observed in WT and VC after $8 \mathrm{~d}$ of treatment (Fig. 3a). A dehydration assay indicated that TaNAC29 transgenic lines exhibited enhanced tolerance on 1/2 MS medium containing $400 \mathrm{mM}$ Mannitol, at both 8 and $16 \mathrm{~d}$ after treatment (Fig. 3b). These results further demonstrated that TaNAC29- 

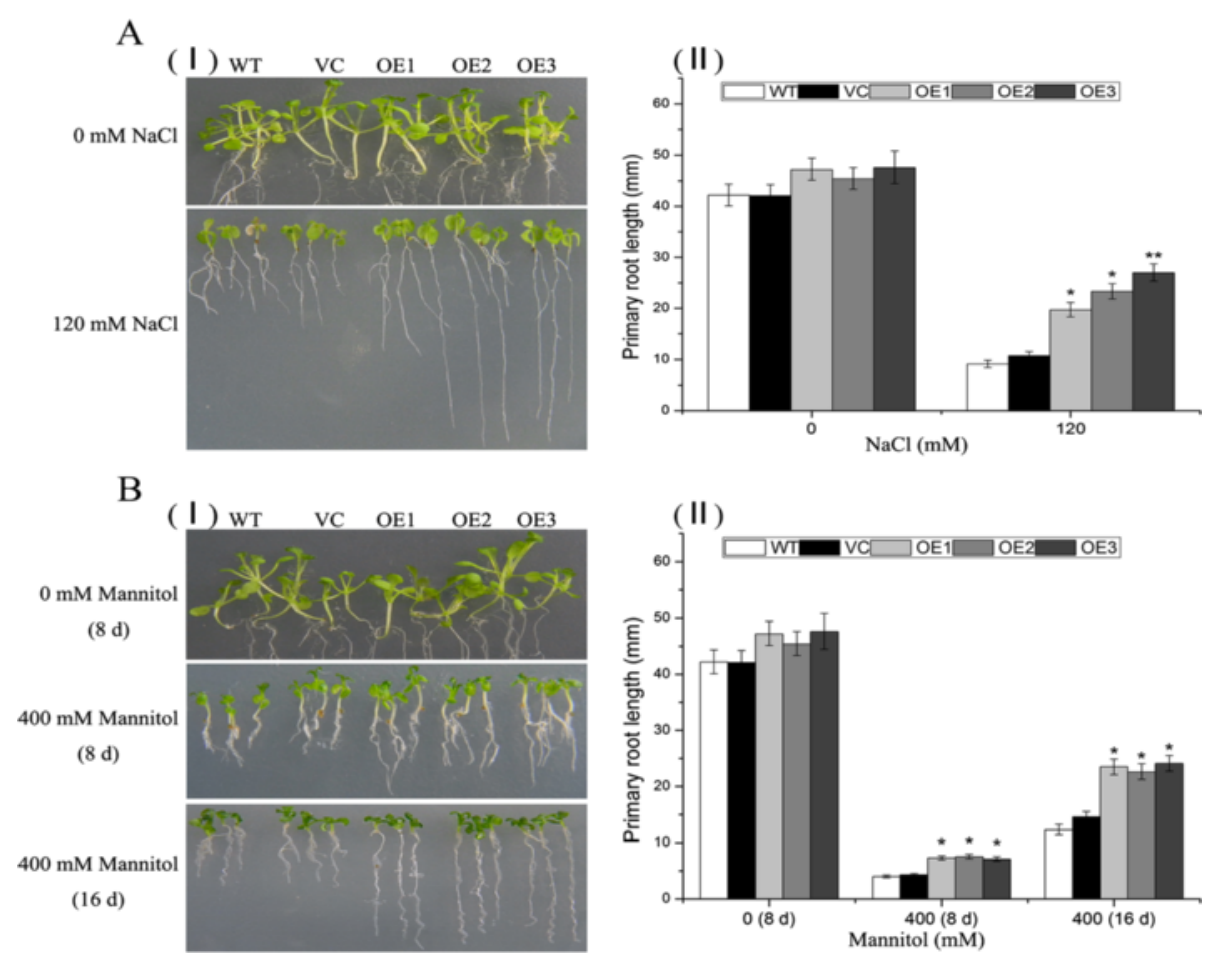

Fig. 3 Root length assays of WT, VC (vector control) and overexpression (OE) lines. a Phenotypes of WT, VC and OE plants grown for $8 \mathrm{~d}$ on medium supplemented with 0 or $120 \mathrm{mM} \mathrm{NaCl}(\mathrm{I})$ and primary root length (II). b Phenotypes of WT, VC and OE plants grown for 8 and $16 \mathrm{~d}$ on medium supplemented with 0 or $400 \mathrm{mM}$ Mannitol (I) and primary root length (II). Values are means \pm SE ( $n=20$ to 25 plants) in root length assays. Asterisks indicate statistically significant differences from $W T\left({ }^{*} P<0.05,{ }^{*} P<0.01\right)$

overexpression lines had increased tolerance to salt and drought stresses.

\section{TaNAC29-overexpressing plants exhibit ABA-hypersensitive response}

Whether salt and drought tolerances of TaNAC29-overexpression line plants was associated with ABA was tested by measuring root length. When grown on $1 / 2 \mathrm{MS}$ medium containing $10 \mu \mathrm{M}$ ABA, exogenous ABA inhibited root growth of TaNAC29-overexpression line seedlings more severely than observed in WT and VC, suggesting that TaNAC29 transgenic lines were hypersensitive to ABA (Fig. 4a). Seed germination and seedling emergence (seedling with cotyledon) rate assays were performed to further verify the ABA hypersensitivity of TaNAC29 transgenic lines. As shown in Fig. 4b-I, when grown on $1 / 2 \mathrm{MS}$ medium without $\mathrm{ABA}$, there was no significant difference in seedling emergence between WT, $\mathrm{VC}$, and transgenic seeds. However, the seedling emergence rate of WT and VC was higher than those of transgenic seeds on $1 / 2$ MS medium containing $2 \mu \mathrm{M} \mathrm{ABA}$ (Fig. 4b I and II). Additionally, transgenic lines grown on ABA-containing medium had shorter roots than those of WT and VC (Fig. 4b-III). These observations indicated that TaNAC29 transgenic lines displayed
ABA hypersensitivity during post-germination growth, suggesting that TaNAC29 was positively regulated by ABA.

\section{Expression of related marker genes under salt and drought stresses}

To further understand the molecular basis of TaNAC29 function, the expression levels of related marker genes were analyzed. The expression levels of most related marker genes were significantly lower in TaNAC29overexpression line plants than in WT plants (Fig. 5). The transcript level of $R D 29 b$ (responsive-to-desiccation 29b; an ABA-responsive marker gene) [29] increased 72-fold in WT under drought stress conditions, this was much greater than observed in TaNAC29-overexpression line plants (Fig. 5a), indicating that TaNAC29 might participate in the ABA signal pathway. As indicators for leaf senescence, relative expressions of SAG13 (senescence-associated gene 13) [30] and SAG113 (senescence-associated gene 113) [31] were lower in TaNAC29-overexpression line plants than in WT plants following salt and drought stresses (Fig. 5b and c), suggesting that TaNAC29 overexpression delayed leaf senescence effects of abiotic stresses. AIB1 (ABA-inducible BHLH-type 


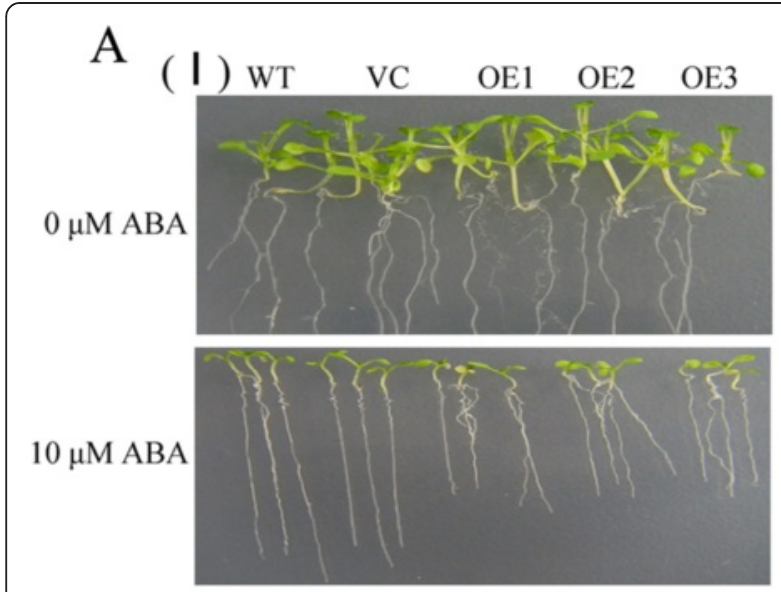

B

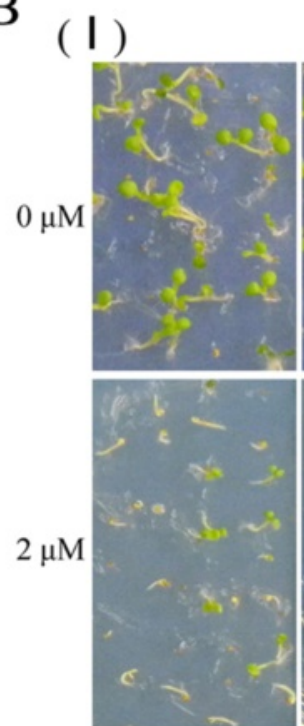

WT
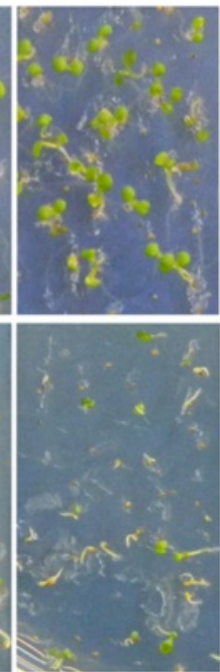

VC
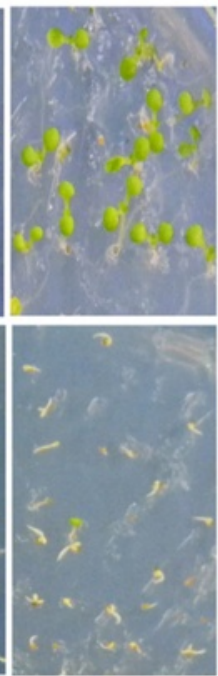

OE1
(II)

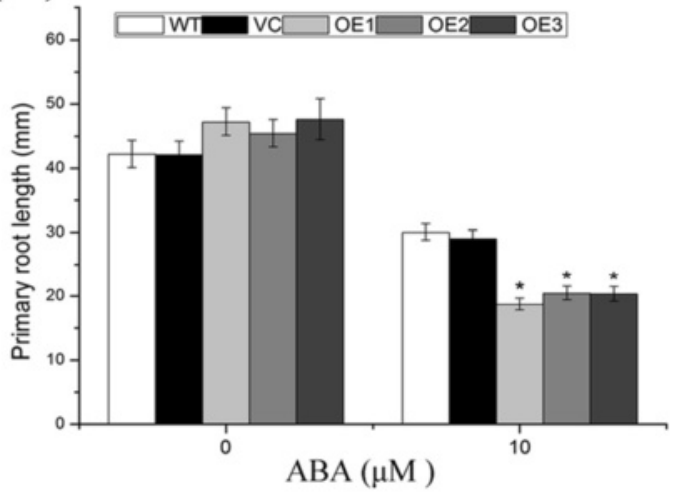

(II)
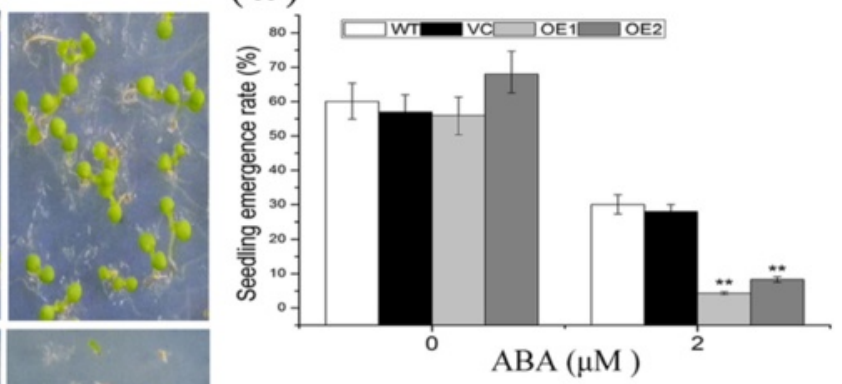

(III) Root length

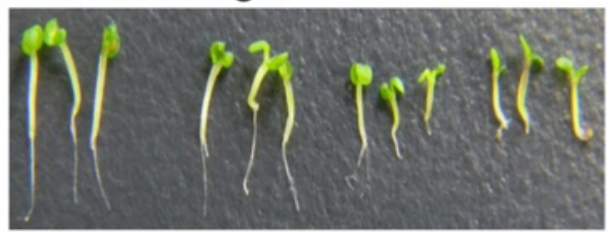

VC

OE1

OE2

Fig. 4 Hypersensitivity of TaNAC29-overexpression (OE) lines to ABA. a Phenotypes of WT, VC (vector control) and OE plants grown for $8 \mathrm{~d}$ on medium supplemented with 0 or $10 \mu \mathrm{M}$ ABA (I) and primary root length (II). Values are means \pm SE ( $n=20$ to 25 plants). Asterisks indicate statistically significant differences from WT ( $\left.{ }^{*} P<0.05\right)$. b Seedlings of WT, VC and TaNAC29-overexpression lines observed $8 \mathrm{~d}$ after germination on $1 / 2$ MS medium supplemented with 0 or $2 \mu \mathrm{M}$ ABA (I) and quantitative analysis of seedling emergence rate (II). Values are means \pm SE ( $n=60$ to 90 seeds). Asterisks indicate statistically significant differences from WT (**P<0.01). Comparison of root length of WT, VC and TaNAC29-overexpression lines (III). Three independent experiments were performed, each evaluating 60 to 90 seeds

transcription factor/JA-associated MYC2-like1), a negative regulator of jasmonic acid (JA) signaling [32], was only slightly affected by salt and drought stresses (Fig. 5d). The expression levels of ERD11 (early-responsive-to-dehydration 11; an early responsiveto-dehydration gene) [28] and ABI5 (ABA-insensitive 5 ; an ABA signaling regulator) [33] significantly increased in WT plants, but were only slightly changed in plants overexpressing TaNAC29 (Fig. 5e and f). Taken together, these results demonstrated that TaNAC29 was involved in regulating the expression of some key ABA signaling regulators and senescenceassociated genes.
Variations of chlorophyll, $\mathrm{H}_{2} \mathrm{O}_{2}$, and malondialdehyde (MDA) content, of electrolytic leakage, and catalase (CAT), superoxide dismutase (SOD), and peroxidase (POD) activities under salt and drought stresses

Abiotic stress can increase the accumulation of reactive oxygen species (ROS), leading to increased oxidative stress [34]. To estimate the level of abiotic stress damage to plant material, related physiological indices in WT and transgenic lines at different time points after stress treatment were measured. Under salt stress, the decrease in chlorophyll content was greater in WT plants than in TaNAC29-overexpression lines (Fig. 6a). There was no significant difference in $\mathrm{H}_{2} \mathrm{O}_{2}$ accumulation, relative 


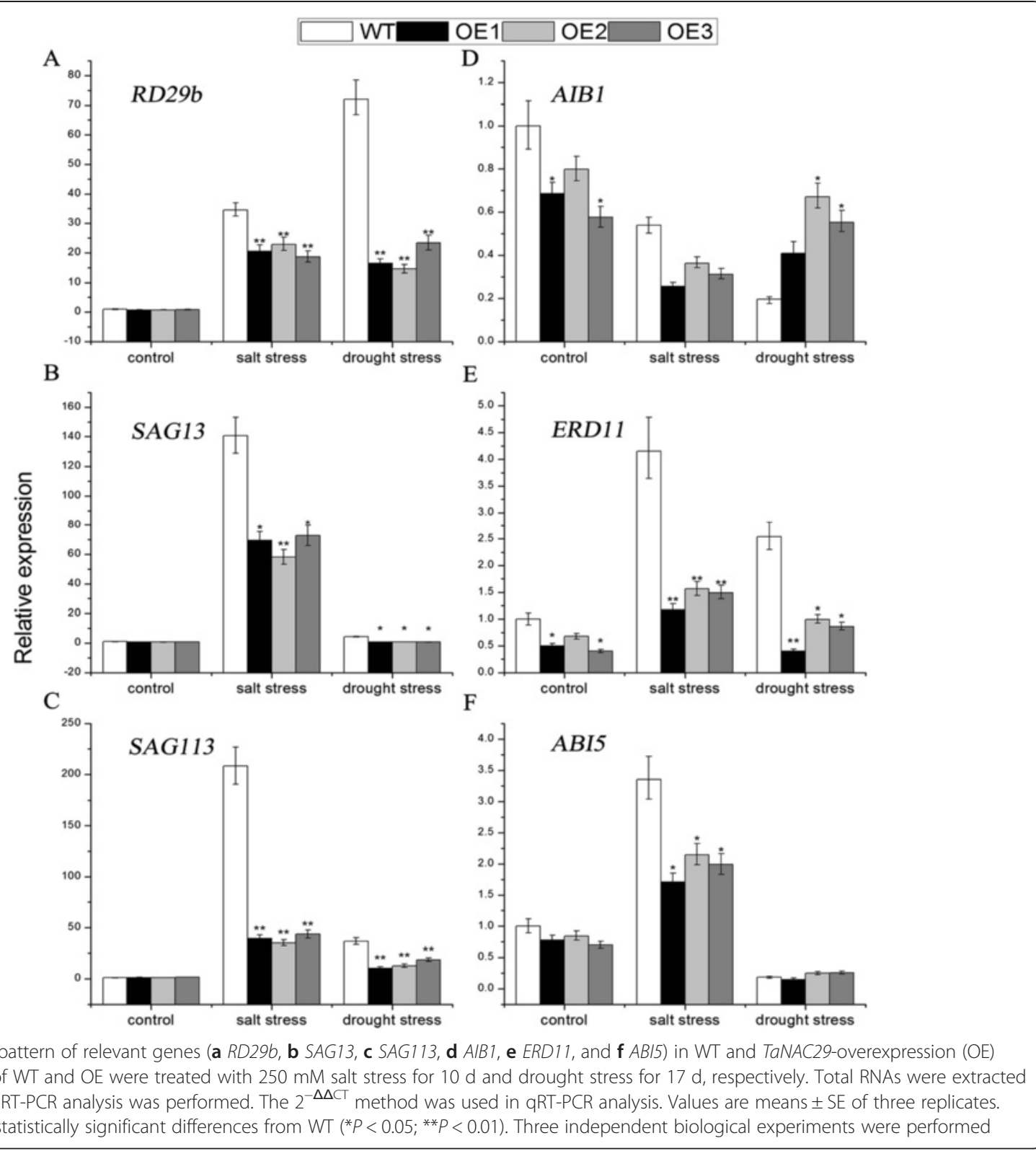

electrolytic leakage, and MDA content between WT plants and TaNAC29-overexpressing lines under normal conditions (Figs. 6 and 7). However, under salt and drought stresses, WT plants showed a greater accumulation of $\mathrm{H}_{2} \mathrm{O}_{2}$ than transgenic lines at all time points. This demonstrated that WT plants were more seriously damaged, and overexpression of TaNAC29 protected transgenic lines from abiotic stress. The electrolytic leakage of the WT plants and TaNAC29overexpression lines increased significantly, though the increment of the WT was higher than observed in transgenic lines, suggesting increased membrane damage might lead to increased solute leakage. MDA content was significantly lower in TaNAC29-overexpression lines than in WT plants under stress conditions, indicating that the transgenic plants produced less ROS.

To further estimate antioxidant enzyme activities, the relative activities of CAT, SOD, and POD [35] in WT and TaNAC29-overexpression lines were measured at different time points following stress treatments. The enzyme activity of SOD in TaNAC29-overexpression plants was significantly higher than in WT plants after subjection to salt and drought stresses (Figs. 6e and 7d), suggesting more superoxide radicals were converted into $\mathrm{O}_{2}$ and $\mathrm{H}_{2} \mathrm{O}_{2}$ via SOD catalysis in transgenic plants [35]. CAT is an important antioxidant enzyme involved in $\mathrm{H}_{2} \mathrm{O}_{2}$ detoxification [35], levels decreased significantly in WT plants but only slightly changed in TaNAC29- 


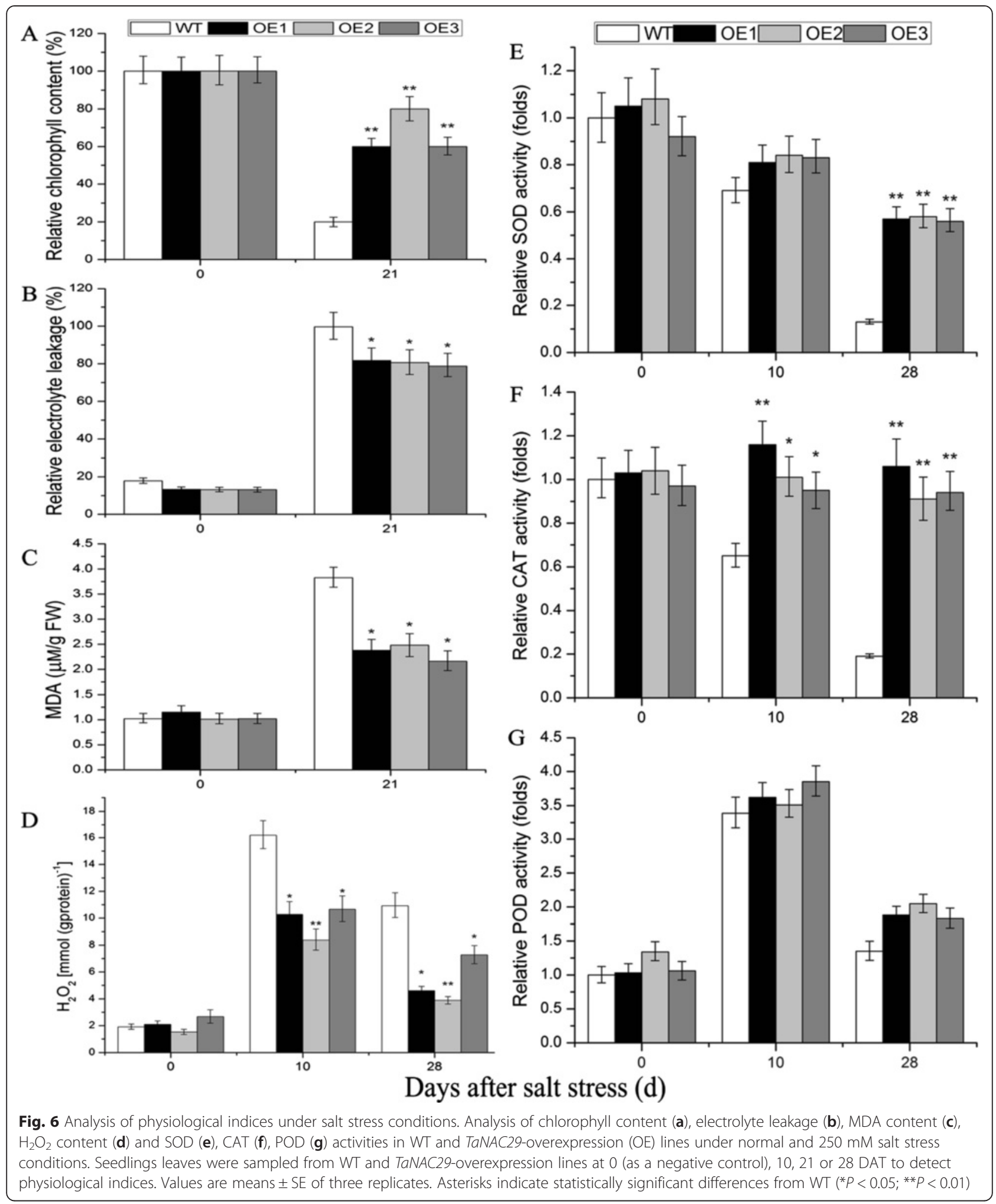

overexpression lines following salt stress (Fig. 6f), indicating that TaNAC29-overexpression lines scavenged more $\mathrm{H}_{2} \mathrm{O}_{2}$. POD activity in WT plants and TaNAC29overexpression lines significantly increased, yet the increment had no significant difference after salt and drought stresses (Figs. 6g and 7f). The higher antioxidant activity of enzymes in transgenic plants may lead to greater scavenging of ROS, thus increasing the survival rates of the 


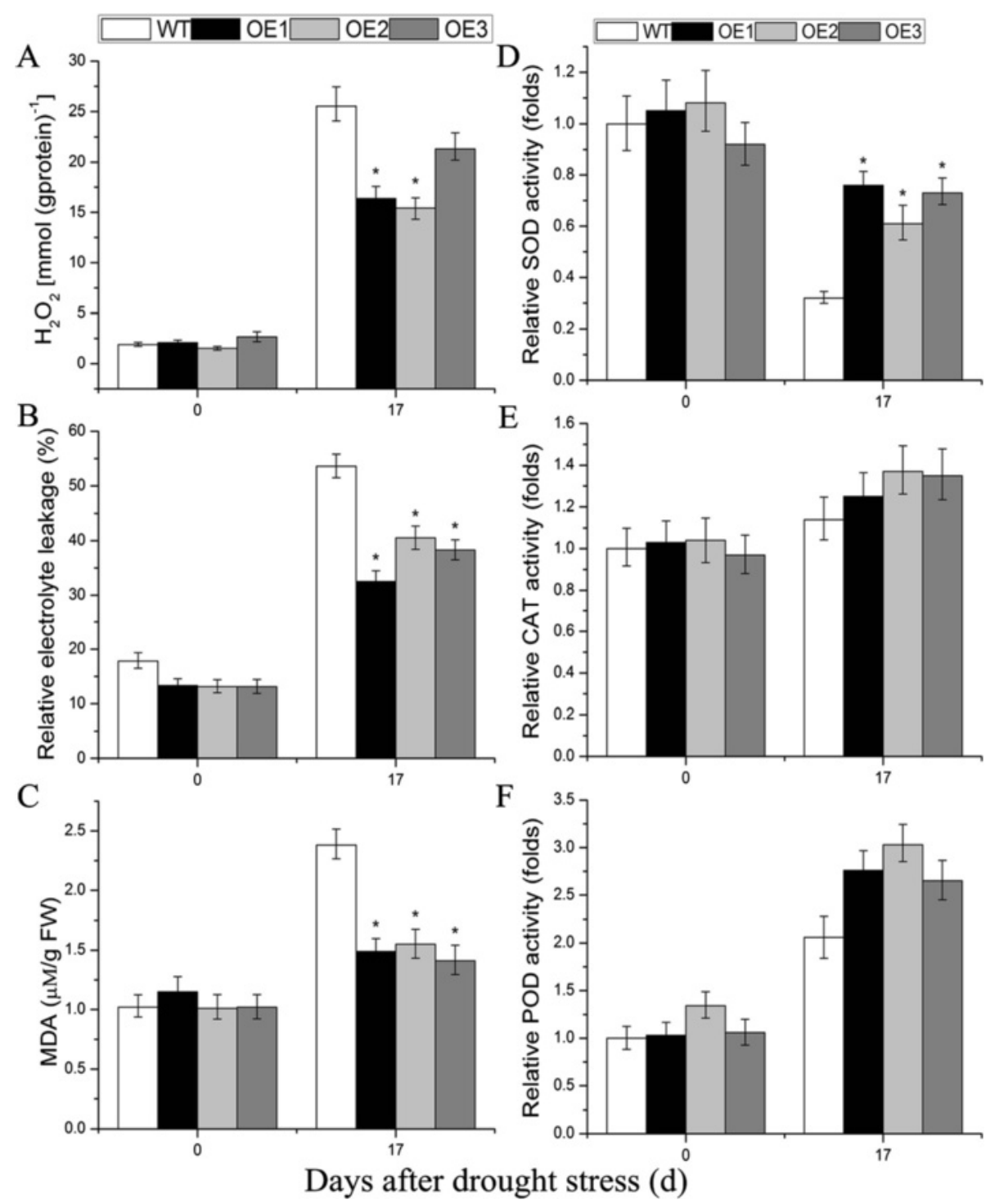

Fig. 7 Analysis of physiological indices under drought stress conditions. Analysis of $\mathrm{H}_{2} \mathrm{O}_{2}$ content (a), electrolyte leakage (b), MDA content (c) and SOD (d), CAT (e), POD (f) activities in WT and TaNAC29-overexpression (OE) lines under normal and drought stress conditions. Seedlings leaves were sampled from WT and TaNAC29-overexpression lines at 0 (as a negative control) and 17 DAT to detect physiological indices. Values are means \pm SE of three replicates. Asterisks indicate statistically significant differences from WT $\left({ }^{*} P<0.05\right)$

transgenic plants $[34,35]$. These results demonstrated that TaNAC29-overexpression lines increased resistance to salt and drought stresses through greater scavenging of ROS.

\section{Discussion}

TaNAC29 plays important roles in abiotic stress response and senescence

The TaNAC29-overexpression plants had significantly increased tolerance to salt and drought stresses. Numerous studies show that NAC TFs play critical roles in the response to biotic and abiotic stresses [3]. ATAF1, ANAC019, ANAC055, and RD26 all enhance tolerance to drought stress [4, 5]. Overexpression of SNAC1,
OsNAC5, OsNAC9, OsNAC6, OsNAC10, and OsNAC45 results in enhanced tolerance to abiotic stresses [9-15]. TaNAC69 confers significant enhancement of drought tolerance in transgenic wheat [18]. Overexpression of TaNAC2, TaNAC2a, and TaNAC67 in transgenic plants improves tolerance to multiple abiotic stresses [19-21]. The expression level of TaNAC29 was much higher in senescent leaves, indicating that TaNAC29 maybe also involved in leaf senescence. In Arabidopsis, ANAC016, AtNAP/ANAC029, ATAF1, and ORE1/ANAC092 act as positive regulators of leaf senescence [30, 36-39], whereas, JUB1 and VNI2 act as negative regulators of leaf senescence $[7,8]$; plants overexpressing these genes 
exhibit a delayed senescence phenotype $[7,8]$, similar to the TaNAC29-overexpression lines. Interestingly, these senescence associated NAC TFs are also involved in abiotic stresses. ANAC016-overexpression plants have low drought tolerance [40], and JUB1-overexpression lines have enhanced tolerance to salt and $\mathrm{H}_{2} \mathrm{O}_{2}$-induced oxidative stresses [7]. Thus, these senescence associated NAC TFs play important roles in the crosstalk between leaf senescence and the abiotic stress response, and it is likely that TaNAC29 has a similar role in wheat.

The overexpression of numerous genes enhances tolerance to abiotic stresses; and overexpression of key genes regulates the relative expression levels of other marker genes [33]. $R D 29 b$ is an ABA-responsive marker gene in the SnRK2s pathway, and is induced by abiotic stress $[29,41]$. Lu et al. [42] demonstrated that 35S-ABI1 transgenic Arabidopsis plants grow better than WT under high $\mathrm{C} /$ low $\mathrm{N}$ stress conditions, while $R D 29 b$ relative expression in transgenic Arabidopsis is significantly lower than observed in WT plants under high C/low C conditions. Yang et al. [8] demonstrated that RD29b transcript levels gradually increased during leaf senescence, similar to VNI2 expression. Our results of RD29b relative expression are similar to these reports. SAG113 expression levels were significantly reduced in TaNAC29-overexpressing plants, suggesting that TaNAC29 is involved in the regulation of SAG113 expression. Compared with WT plants, SAG113 knockout plants show less water loss, more sensitive to ABA, delayed leaf senescence, and enhanced tolerance to drought stress [31]; this is similar to the TaNAC29-overexpression line plants. Thus, downregulation of SAG113 expression in plants overexpressing TaNAC29 likely contributed to the enhanced salt and drought stresses tolerance observed.

\section{TaNAC29 participates in the ABA-mediated pathway in stress tolerance}

$\mathrm{ABA}$ is an important signaling molecule involved in plant responses to many unfavorable environmental stresses, including high salinity, drought, and extreme temperature [33], Indeed, ABA is often considered as the second messenger. Our results demonstrated that plants overexpressing TaNAC29 had enhanced tolerance to salt and drought, and exhibited hypersensitivity to ABA. This increased sensitivity to $\mathrm{ABA}$ might be a result of lower transpiration rates and faster stomatal closure in TaNAC29-overexpression plants, leading to lower water loss and enhanced tolerance. A close relationship between ABA-hypersensitivity and abiotic stress tolerance has been demonstrated. For example, overexpression of $\mathrm{K}^{+}$uptake transporter 6 (KUP6) in Arabidopsis exhibits increased ABA sensitivity through faster stomatal closure and enhanced tolerance to drought stress [43]. Conversely, mutation in Arabidopsis more axillary growth 2 (MAX2), MAX3, and MAX4 genes results in reduced ABA sensitivity, impaired ABA-mediated stomatal closing, and decreased survival rates under drought stress [44]. Numerous studies have revealed how abiotic stresses trigger signal transductions necessary for plant survival via ABA and other phytohormone signal molecules. In Arabidopsis, ATAF1 acts as a positive regulator of ABA biosynthesis [45]. Conversely, ATAF2 expression is induced by methyl jasmonate (MeJA) and salicylic acid, but is independent of ABA signaling [6]. Plants overexpressing ATAF1, ANAC019, RD26, and OsNAP show enhanced tolerance to drought, but increased sensitivity to ABA $[5,28,46,47]$. Overexpression of ANAC019 and ANAC055 increases JA-induced expression of defense genes [48]. NTM2/ANAC069-mediated salt signaling in seed germination is not related to ABA and gibberellin (GA), meaning NTM2 acts independently of the ABA signal [49]. VNI2 is induced by high salinity in an ABA-dependent manner; this gene delays leaf aging and senescence [8]. Thus, many genes participating in the ABA signaling transduction network, such as PP2Cs, SnRK2s, NAC, and WRKY, are responsive to external influences [33]. Hence, the ABA hypersensitivity of TaNAC29 suggests that TaNAC29 is involved in cellular network cross-talk between the ABA signal pathway and the abiotic stress-induced metabolic pathway.

\section{The antioxidant mechanism is involved in TaNAC29 conferring salt and drought stresses tolerance}

Abiotic stress can lead to oxidation damage and membrane lipid peroxidation in plants [34, 35]. Therefore, the $\mathrm{H}_{2} \mathrm{O}_{2}$ content often increases under stress conditions. In Arabidopsis, $\mathrm{H}_{2} \mathrm{O}_{2}$ treatment increases JUB1 and ATAF1 transcript abundance [7, 30]. Plants overexpressing JUB1 counteract cellular accumulation of $\mathrm{H}_{2} \mathrm{O}_{2}$, having decreased $\mathrm{H}_{2} \mathrm{O}_{2}$ levels and an enhanced tolerance to salt stress [7]. In contrast, ATAF1-overexpression plants accumulate significantly higher $\mathrm{H}_{2} \mathrm{O}_{2}$ than WT plants; ataf1 mutant plants accumulate less $\mathrm{H}_{2} \mathrm{O}_{2}$ and display delayed senescence [30]. TaNAC29-overexpression plants also accumulated less $\mathrm{H}_{2} \mathrm{O}_{2}$ and had enhanced tolerance to salt and drought stresses. MDA is often increased under stress conditions, and ROS can be produced by membrane lipid peroxidation thus affecting protein synthesis and stability in plants [34, 35]. Hence, the extent of membrane lipid peroxidation was used to assess the severity of oxidation stress. Antioxidant enzymes such as CAT, SOD, and POD play an important role in scavenging $\operatorname{ROS}[34,35]$. These antioxidant enzymes are often upregulated to defend protein systems after stress, however, several studies have demonstrated that activities of these antioxidant enzymes decline under salt and drought stresses over long time treatment conditions, suggesting that the defense mechanism of these 
antioxidant enzymes may have been destroyed [34, 35]. Thus, together with the higher enzyme activities of SOD or CAT (Figs. 6 and 7), most TaNAC29-overexpression plants still survived under salt and drought stresses conditions.

\section{Conclusions}

In this study, TaNAC29 was upregulated by various abiotic stresses, and played important roles in senescence and tolerance to high salinity and drought. It was demonstrated that TaNAC29 participates in the ABAmediated pathway, and activates antioxidant enzymes to improve plant tolerances. These findings shed some light on the complex mechanisms and role of NAC in a plant's response to environmental stresses.

\section{Methods}

\section{Plant materials and stress treatments}

Bread wheat (Triticum aestivum L. cv. Chinese spring) was used in this study. Seeds were germinated in water and cultivated under a 12-h light/12-h dark cycle at $22{ }^{\circ} \mathrm{C}$ in a greenhouse. Fourteen-day-old wheat seedlings were treated by different stresses or signaling molecules. Multiple abiotic stress treatments were performed by submerging wheat seedling roots in solutions of $200 \mathrm{mM} \mathrm{NaCl}$, $20 \% \mathrm{PEG} 6000$, and $10 \mathrm{mM} \mathrm{H}_{2} \mathrm{O}_{2}$, respectively. For phytohormone treatments, wheat seedling leaves were sprayed with $100 \mu \mathrm{M}$ ABA, meanwhile wheat seedling roots were submerged in the same concentration of ABA solution. Wheat seedlings treated with water were used as a mock control. Leaves and roots of wheat seedlings were sampled at different time-points $(0,1,3,6,12$, and 24 h). Field grown wheat plants were used for measuring organspecific expression patterns of TaNAC29. Young root, stem, and leaf (at the four-leaf stage), mature root, stem, and leaf (at anthesis stage), flag leaf, stamen, pistil, embryo, endosperm, coleoptile, and caryopsis were collected. All collected samples were immediately frozen in liquid nitrogen and stored at $-80{ }^{\circ} \mathrm{C}$ for future analysis.

\section{Cloning and bioinformatic analysis of TaNAC29}

To identify novel $N A C$ genes in wheat, in silico cloning was used to predict putative TaNAC genes. Previously, Jeong et al. [11] reported that OsNAC10-overexpression rice plants had enhanced tolerance to drought stress. Therefore, the barley (Hordeum vulgare) gene HvNACO23 (GenBank: CBZ41159.1), with high homology to OsNAC10, was used as a query probe to blast the EST library of wheat (http://www.ncbi.nlm.nih.gov/nucest/?term=Triticum+aesti vum). Some highly homologous EST sequences were obtained, and assembled using the CAP3 program (http:// doua.prabi.fr/software/cap3). Next, the predicted NAC-like gene was used as a query probe to blast the EST library of wheat again, thus obtaining a longer sequence. After repeats for several times, a full-length cDNA sequence, including ORF, 5'-UTR, and 3'-UTR regions was obtained. Next, primer pairs (Additional file 2: Table S1a) were designed to amplify this putative sequence from cDNA templates synthesized from RNAs mixtures extracted from different wheat organs and under various stress conditions (see above). The polymerase chain reaction (PCR) product was cloned into a pMD18-T vector (TaKaRa, Dalian, China) and transfected into E. coli TOP 10 competent cells (Tiangen, Beijing, China). Finally, the target gene in positive cloned strains was sequenced (AuGCT Biotech, Beijing, China). The cloned NAC gene, designated TaNAC29 (GenBank: KT783450), was analyzed using online software available at the InterProScan website (http://www.ebi.ac.uk/Tools/pfa/iprscan5/). The conserved NAC domain at the N-terminal, and the L motif (also named LP motif) $[27,28]$ at the C-terminal, were searched using MEME (http://memesuite.org/tools/meme). A prediction of structural ID was performed using PONDR VL3 (http://www.pondr.com). Alignment of relevant sequences was performed using MEGA software (version 5.1) and MegAlign of DNAStar.

\section{qRT-PCR analysis of TaNAC29 expression patterns}

Total RNA was extracted from different organs and various stress treated materials (see above) using Trizol in accordance with manufacturer's instructions (Zomanbio, Beijing, China). Integrity and quality of total RNA samples were examined by electrophoreses by running $5 \mu \mathrm{l}$ RNA in an ethidium bromide agarose gel. First-strand cDNA synthesis was performed according to the FastQuant RT kit protocol (Tiangen). Organ-specific expression patterns and gene expression patterns of TaNAC29, after various stress treatments, were determined by qRT-PCR analysis. Gene specific primer pairs (Additional file 2: Table S1b) were designed at the 3 '-UTR region of the nucleic acid sequence, thus excluding the NAC conserved domain. To test primer accuracy and specificity, PCR was performed and the amplified product was confirmed by sequencing. TaActin (GenBank: AB181991.1; Additional file 2: Table S1b) was used as the internal reference gene when examining gene expression of TaNAC29. qRT-PCR analysis was performed using a Biorad CFX system (Bio-Rad Laboratories, Hercules, CA). During the qRT-PCR analysis, each sample was analyzed using three technical replicates, and data analyzed by analysis software based on the comparative $2^{-\Delta \Delta \mathrm{CT}}$ method of relative gene quantification [50]. Relative expression of TaNAC29 and probability value $(P)$ were also calculated using the qRT-PCR analysis software.

\section{Subcellular localization of TaNAC29 in wheat protoplasts} To investigate the subcellular localization of TaNAC29, a recombinant construct of TaNAC29-GFP was transformed 
into wheat mesophyll protoplasts using the DNA-PEGcalcium transfection method [51]. To obtain high concentration recombinant plasmids, the pMD18-T plasmid was fused with cauliflower mosaic virus $35 \mathrm{~S}$ promoter and GFP sequence, resulting in the transformation plasmid pMD18-35S-GFP vector. The TaNAC29 ORF (excluding

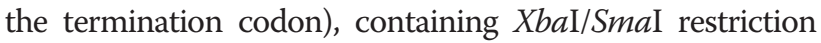
sites, was amplified using specific primer pairs (Additional file 2: Table S1c). The PCR product was then inserted into the XbaI/SmaI sites of the pMD18-35S-GFP vector. Subsequently, 35S::TaNAC29-GFP fusion protein construct, and the pMD18-35S-GFP vector as a negative control, were separately introduced into wheat mesophyll protoplasts using the DNA-PEG-calcium transfection method [51]. Finally, transformed mesophyll protoplasts were examined by fluorescence microscopy (OLYMPUS DP72, Japan) after incubation at $20{ }^{\circ} \mathrm{C}$ for $20 \mathrm{~h}$ in the dark. The specific nuclear stain DAPI (Beyotime Biotech, Jiangsu, China) was used for observing transformed mesophyll protoplasts.

\section{Transactivation activity analysis of TaNAC29 in yeast}

Transactivation activity analysis was performed to examine the expressions of HIS3, ADE2, and LacZ reporter genes in the yeast strain $A H 109$. The full-length ORF

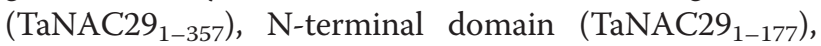
C-terminal regulatory (TaNAC29 ${ }_{178-357}$ ), TaNAC29 $1_{1-233}$ without L motif and six truncated versions (TaNAC29 261. TaNAC291-250, TaNAC29 ${ }_{178-242}$, TaNAC29 $243-273$, TaNAC29 $274-312$, and TaNAC29 $313-357$ ) with particular L motif sequences [27] were amplified by PCR using specific primers containing EcoR1/BamH1 restriction sites (Additional file 2: Table S1d). Each PCR product was inserted into EcoR1/BamH1 sites of the pGBKT7 vector (Clontech, Palo Alto, CA). Subsequently, the ten constructs and negative control pGBKT7 vector were transformed into yeast strain AH109 (Clontech). The transformed yeast cultures were plated onto synthetic dropout (SD) plates (-Trp) and SD plates (-Trp/-His/-Ade) for $4 \mathrm{~d}$ at $30{ }^{\circ} \mathrm{C}$, before detection of transactivation property. For easy evaluation of transactivation activity, transformants were dissolved in sterile distilled water and 10-fold serial dilutions prepared. Finally, $3 \mu \mathrm{l}$ of each dilution was spotted onto SD plates (-Trp/-His/-Ade) and SD plates (-Trp/-His) with X$\alpha$-Gal (Clontech) for $4 \mathrm{~d}$ at $30^{\circ} \mathrm{C}$.

Plant transformation and generation of transgenic plants

To obtain transgenic Arabidopsis plants, the coding sequence of the TaNAC29 containing termination codon was amplified by RT-PCR and cloned into $\mathrm{XbaI} / \mathrm{SmaI}$ restriction sites of the pBI121 vector (Clontech) under the control of the $35 \mathrm{~S}$ promoter of cauliflower mosaic virus. The primers containing the $\mathrm{XbaI} / \mathrm{SmaI}$ restriction sites are listed in Additional file 2: Table S1e. The recombinant vector pBI121-TaNAC29 and the pBI121 empty VC were introduced into Agrobacterium tumefaciens strain EHA105 (Biovector Science Lab, Inc., Beijing, China). Finally, transgenic Arabidopsis plants were generated using the A. tumefaciens-mediated floral dipping method [52]. For generation of homozygous progenies, $\mathrm{T}_{1}$ and $\mathrm{T}_{2}$ seeds were screened on kanamycin $(50 \mathrm{mg} / \mathrm{L})$ plates for selection. Homozygous $\mathrm{T}_{3}$ progenies were confirmed by PCR analysis, and TaNAC29 expression levels were examined by semi-quantitative analysis using gene-specific primers (Additional file 2: Table S1e). Representative lines overexpressing TaNAC29 were used for further analysis.

\section{Stress tolerance assays of TaNAC29-overexpression plants}

The WT, VC, and TaNAC29-overexpression lines were used to evaluate various stress tolerances. After stratification at $4{ }^{\circ} \mathrm{C}$ for $3 \mathrm{~d}$, Arabidopsis seeds were germinated on $1 / 2$ MS medium ( $1 \%$ sucrose, no vitamins) under a 16-h light/8-h dark cycle at $22{ }^{\circ} \mathrm{C}$ in a growth chamber for an additional $4 \mathrm{~d}$. Four-day-old seedlings were transferred to $1 / 2 \mathrm{MS}$ medium supplemented with $10 \mu \mathrm{M}$ ABA for signal molecule response assay, and with $120 \mathrm{mM} \mathrm{NaCl}$ or $400 \mathrm{mM}$ Mannitol for osmotic stress assays. Root length was scored after 8 or $16 \mathrm{~d}$ of growth on vertical plates. To conduct seedling emergence rate assays for inspection of the ABA response, Arabidopsis seeds were sown on 1/2 MS medium supplemented with 0 or $2 \mu \mathrm{M}$ ABA. Seedling emergence rates (seedling with cotyledon) were scored on plates after $8 \mathrm{~d}$ growth.

To obtain full-grown Arabidopsis plants, WT, VC, and TaNAC29-overexpression plants were grown in plastic containers filled with humus soil and cultivated in a greenhouse with a 16 -h light $\left(80\right.$ or $\left.120 \mu \mathrm{mol} \mathrm{m}{ }^{-2} \mathrm{~s}^{-1}\right) / 8$-h dark cycle at $22{ }^{\circ} \mathrm{C}$. For the water loss assay, rosette leaves were detached from 25-day-old seedlings and placed on filter paper for water deficiency treatment at $25{ }^{\circ} \mathrm{C}$. Meanwhile changes in rosette leaf shape were observed during the airdry treatment. The weight of rosette leaves was measured at 1-h intervals. Drought tolerance assays were performed, at different growth periods (25-, and 65-day-old), using Arabidopsis plants cultured in a greenhouse without watering. For the salt tolerance assay, Arabidopsis plants grown at different growth periods (25-, 45-, and 65-day-old) were subjected to $250 \mathrm{mM} \mathrm{NaCl}$ treatment for 21 or $28 \mathrm{~d}$ after withholding of water for $2 \mathrm{w}$.

\section{Expression analyses of relevant marker genes}

To detect expression of relevant marker genes in WT and TaNAC29-overexpression plants under salt and drought stresses, leaves of Arabidopsis seedlings were detached after subjection to $250 \mathrm{mM}$ salt stress for $10 \mathrm{~d}$ or drought stress for $17 \mathrm{~d}$. Total RNA was extracted from leaves using Trizol in accordance with manufacturer's instructions (Zomanbio, Beijing, China), and qRT-PCR analysis performed to examine marker gene expressions of 
RD29b (At5g52300), ERD11 (At1g02930), AIB1 (At2g46 510), ABI5 (At2g36270), SAG13 (At2g29350), and SAG113 (At5g59220); Actin2 was used as the internal control gene. Three independent biological experiments were performed. Primer pairs used are listed in Additional file 2: Table S1f.

\section{Measurement of electrolytic leakage, of chlorophyll, MDA, and $\mathrm{H}_{2} \mathrm{O}_{2}$ content, and of SOD, CAT, and POD activity}

To detect changes in physiological indices under salt and drought stress conditions, leaves were collected from plants at different time points $(0,10,17,21$, and $28 \mathrm{~d})$ during stress treatments. Electrolytic leakage, the contents of chlorophyll, $\mathrm{MDA}$, and $\mathrm{H}_{2} \mathrm{O}_{2}$, and the enzyme activities of SOD, CAT, and POD were measured. For extraction of $\mathrm{H}_{2} \mathrm{O}_{2}, \mathrm{CAT}$, SOD, and POD, $0.2 \mathrm{~g}$ of leaves were sampled with $1.8 \mathrm{ml}$ phosphate-buffered saline (PBS; $0.1 \mathrm{~mol} / \mathrm{L}, \mathrm{pH} 7.4$ ) on ice. The crude extract was centrifuged at $10,000 \mathrm{~g}$ for $10 \mathrm{~min}$ at $4{ }^{\circ} \mathrm{C}$. The $\mathrm{H}_{2} \mathrm{O}_{2}$ content, and CAT, SOD, and POD enzyme activities in the supernatant were immediately measured by enzyme-linked immunosorbent assay (ELISA), using the corresponding detection kits (A064-1, A007-1, A001-1, and A084-3; Jiancheng, China). MDA content analysis was performed using the thiobarbituric acid method [53]. Chlorophyll was extracted and examined using the protocol [54]. Electrolyte leakage was determined by relative conductivity as described by $\mathrm{Hu}[55]$.

\section{Availability of supporting data}

All the supporting data are included as additional files.

\section{Additional files}

Additional file 1: Figure S1. Structural features of TaNAC29 protein. Figure S2. Phylogenetic relationship of TaNAC29 with other NAC members. Figure S3. Alignment of the nucleic acid sequences. Figure S4. Transactivation activity analysis of TaNAC29 protein in yeast. Figure S5. Subcellular localization of TaNAC29. Figure S6. Organ-specific expression patterns of TaNAC29 in wheat. Figure S7. Expression of TaNAC29 in transgenic Arabidopsis lines. Figure S8. Delaying in bolting and flowering of TaNAC29-overexpression line 1 (OE1) in comparison to the WT plants. Figure S9. TaNAC29 overexpression (OE) lines exhibit enhanced tolerance to water deficiency. Figure S10. Root growth of WT and TaNAC29-overexpression (OE) lines in response to drought stress. (PDF $1392 \mathrm{~kb}$ )

Additional file 2: Table S1. Primers used in amplification of DNA sequences. (PDF $83 \mathrm{~kb}$ )

\section{Abbreviations}

ABA: Abscisic acid; ABI5: ABA-insensitive 5; AlB1: ABA-inducible BHLH-type transcription factor/JA-associated MYC2-like1; CAT: Catalase; d: Day; DAPI: 4',6-diamidino-2-phenylindole; ELISA: Enzyme-linked immunosorbent assay; ERD 11: Early-responsive-to-dehydration 11; GA: Gibberellin; GFP: Green fluorescent protein; ID: Intrinsically disordered; JA: Jasmonic acid; KUP6: $K^{+}$ uptake transporter 6; MAX2: More axillary growth 2; MDA: Malondialdehyde; MeJA: Methyl jasmonate; MEME: Multiple EM for motif elicitation; MS: Murashige-Skoog; NAC: NAM, ATAF and CUC; OE: Overexpression; ORF: Open reading frame; $P$ : Probability value; PBS: Phosphate-buffered saline; PCR: Polymerase chain reaction; POD: Peroxidase; qRT-

PCR: Quantitative real-time polymerase chain reaction; RD29b: Responsive-todesiccation 29b; ROS: Reactive oxygen species; SAG13: Senescence-associated
}

gene 13; SAG113: Senescence-associated gene 113; SD: Synthetic dropout; SOD: Superoxide dismutase; TFs: Transcription factors; UTR: Untranslated region; VC: Vector control; w: Week; WT: Wild type.

\section{Competing interests}

The authors declare that they have no competing interests.

\section{Authors' contributions}

GYH and GXY designed the study and participated in paper writing. QJH conducted all experiments, analyzed the data and drafted the manuscript. YW helped to conduct experiments. BL, JLC, MJC and KXL participated in transactivation activity assays. All authors read and approved the final manuscript.

\section{Authors' information}

Not applicable.

\section{Availability of data and materials}

Not applicable.

\section{Funding}

This work was supported by National Genetically Modified New Varieties of Major Projects of China (2015ZX08002-004, 2015ZX08010-004); International S \& T Cooperation Key Projects of Chinese Ministry of Science and Technology (grant No. 2009DFB30340); Research Fund for the Doctoral Program of Higher Education of China (Grant no. 2012014211075), Open Research Fund of State Key Laboratory of Hybrid Rice in Wuhan University in China (Grant no. KF201302)

Received: 30 July 2015 Accepted: 12 October 2015

Published online: 04 November 2015

\section{References}

1. Cramer GR, Urano K, Delrot S, Pezzotti M, Shinozaki K. Effects of abiotic stress on plants: a systems biology perspective. BMC Plant Biol. 2011;1 1:163.

2. Puranik S, Sahu PP, Srivastava PS, Prasad M. NAC proteins: regulation and role in stress tolerance. Trends Plant Sci. 2012:17(6):369-81.

3. Nakashima K, Takasaki H, Mizoi J, Shinozaki K, Yamaguchi-Shinozaki K. NAC transcription factors in plant abiotic stress responses. Biochim Biophys Acta. 2012;1819(2):97-103.

4. Tran LS, Nakashima K, Sakuma Y, Simpson SD, Fujita Y, Maruyama K, et al. Isolation and functional analysis of Arabidopsis stress-inducible NAC transcription factors that bind to a drought-responsive cis-element in the early responsive to dehydration stress 1 promoter. Plant Cell. 2004;16(9):2481-98.

5. Wu Y, Deng Z, Lai J, Zhang Y, Yang C, Yin B, et al. Dual function of Arabidopsis ATAF1 in abiotic and biotic stress responses. Cell Res. 2009;19(11):1279-90.

6. Delessert C, Kazan K, Wilson IW, Van Der Straeten D, Manners J, Dennis ES, et al. The transcription factor ATAF2 represses the expression of pathogenesis-related genes in Arabidopsis. Plant J. 2005:43(5):745-57.

7. Wu A, Allu AD, Garapati P, Siddiqui H, Dortay H, Zanor MI, et al. JUNGBRUNNEN1, a reactive oxygen species-responsive NAC transcription factor, regulates longevity in Arabidopsis. Plant Cell. 2012;24(2):482-506.

8. Yang SD, Seo PJ, Yoon HK, Park CM. The Arabidopsis NAC transcription factor VNI2 integrates abscisic acid signals into leaf senescence via the COR/ RD genes. Plant Cell. 2011;23(6):2155-68.

9. Jeong JS, Kim YS, Redillas MC, Jang G, Jung H, Bang SW, et al. OsNAC5 overexpression enlarges root diameter in rice plants leading to enhanced drought tolerance and increased grain yield in the field. Plant Biotechnol J. 2013;11(1):101-14.

10. Redillas MC, Jeong JS, Kim YS, Jung H, Bang SW, Choi YD, et al. The overexpression of OsNAC9 alters the root architecture of rice plants enhancing drought resistance and grain yield under field conditions. Plant Biotechnol J. 2012;10(7):792-805.

11. Jeong JS, Kim YS, Baek KH, Jung H, Ha SH, Do Choi Y, et al. Root-specific expression of OsNAC10 improves drought tolerance and grain yield in rice under field drought conditions. Plant Physiol. 2010;153(1):185-97.

12. Nakashima K, Tran LS, Van Nguyen D, Fujita M, Maruyama K, Todaka D, et al. Functional analysis of a NAC-type transcription factor OsNAC6 involved in abiotic and biotic stress-responsive gene expression in rice. Plant J. 2007;51(4):617-30. 
13. Zheng X, Chen B, Lu G, Han B. Overexpression of a NAC transcription factor enhances rice drought and salt tolerance. Biochem Biophys Res Commun. 2009;379(4):985-9.

14. Hu H, Dai M, Yao J, Xiao B, Li X, Zhang Q, et al. Overexpressing a NAM, ATAF, and CUC (NAC) transcription factor enhances drought resistance and salt tolerance in rice. Proc Natl Acad Sci U S A. 2006;103(35):12987-92.

15. Saad AS, Li X, Li HP, Huang T, Gao CS, Guo MW, et al. A rice stress-responsive NAC gene enhances tolerance of transgenic wheat to drought and salt stresses. Plant Sci. 2013;203-204:33-40.

16. Kaneda T, Taga Y, Takai R, Iwano M, Matsui H, Takayama S, et al. The transcription factor OsNAC4 is a key positive regulator of plant hypersensitive cell death. EMBO J. 2009;28(7):926-36.

17. Sun $L$, Zhang $H$, Li D, Huang $L$, Hong $Y$, Ding $X S$, et al. Functions of rice NAC transcriptional factors, ONAC122 and ONAC131, in defense responses against Magnaporthe grisea. Plant Mol Biol. 2013;81(1-2):41-56.

18. Xue GP, Way HM, Richardson T, Drenth J, Joyce PA, Mclntyre CL. Overexpression of TaNAC69 leads to enhanced transcript levels of stress up-regulated genes and dehydration tolerance in bread wheat. Mol Plant. 2011;4(4):697-712.

19. Mao X, Zhang H, Qian X, Li A, Zhao G, Jing R. TaNAC2, a NAC-type wheat transcription factor conferring enhanced multiple abiotic stress tolerances in Arabidopsis. J Exp Bot. 2012:63(8):2933-46.

20. Tang Y, Liu M, Gao S, Zhang Z, Zhao X, Zhao C, et al. Molecular characterization of novel TaNAC genes in wheat and overexpression of TaNAC2a confers drought tolerance in tobacco. Physiol Plant. 2012;144(3):210-24.

21. Mao X, Chen S, Li A, Zhai C, Jing R. Novel NAC transcription factor TaNAC67 confers enhanced multi-abiotic stress tolerances in Arabidopsis. PLOS ONE. 2014;9(1), e84359.

22. Xia N, Zhang G, Sun Y-F, Zhu L, Xu L-S, Chen X-M, et al. TaNAC8, a novel NAC transcription factor gene in wheat, responds to stripe rust pathogen infection and abiotic stresses. Physiol Mol Plant Pathol. 2010;74(5-6):394-402.

23. Xia N, Zhang G, Liu XY, Deng L, Cai GL, Zhang Y, et al. Characterization of a novel wheat NAC transcription factor gene involved in defense response against stripe rust pathogen infection and abiotic stresses. Mol Biol Rep. 2010;37(8):3703-12.

24. Baloglu MC, Oz MT, Oktem HA, Yucel M. Expression analysis of TaNAC69-1 and TtNAMB-2, wheat NAC family transcription factor genes under abiotic stress conditions in durum wheat (Triticum turgidum). Plant Mol Biol Report. 2012;30(5):1246-52.

25. Jia J, Zhao S, Kong X, Li Y, Zhao G, He W, et al. Aegilops tauschii draft genome sequence reveals a gene repertoire for wheat adaptation. Nature. 2013;496(7443):91-5

26. Rauf M, Arif M, Fisahn J, Xue GP, Balazadeh S, Mueller-Roeber B. NAC transcription factor speedy hyponastic growth regulates flooding-induced leaf movement in Arabidopsis. Plant Cell. 2013;25(12):4941-55.

27. Kjaersgaard T, Jensen MK, Christiansen MW, Gregersen P, Kragelund BB, Skriver K. Senescence-associated barley NAC (NAM, ATAF1,2, CUC) transcription factor interacts with radical-induced cell death 1 through a disordered regulatory domain. J Biol Chem. 2011;286(41):35418-29.

28. Jensen MK, Kjaersgaard T, Nielsen MM, Galberg P, Petersen K, O'Shea C, et al. The Arabidopsis thaliana NAC transcription factor family: structurefunction relationships and determinants of ANAC019 stress signalling. Biochem J. 2010;426(2):183-96.

29. Yamaguchi-Shinozaki K, Shinozaki K. Characterization of the expression of a desiccation-responsive rd29 gene of Arabidopsis thaliana and analysis of its promoter in transgenic plants. Mol Gen Genet. 1993;236:331-40.

30. Garapati P, Xue GP, Munne-Bosch S, Balazadeh S. Transcription factor ATAF1 in Arabidopsis promotes senescence by direct regulation of key chloroplast maintenance and senescence transcriptional cascades. Plant Physiol. 2015;168(3):1122-39

31. Zhang K, Xia X, Zhang Y, Gan S-S. An ABA-regulated and golgi-localized protein phosphatase controls water loss during leaf senescence in Arabidopsis. Plant J. 2012;69:667-78.

32. Nakata M, Mitsuda N, Herde M, Koo AJ, Moreno JE, Suzuki K, et al. A bHLHtype transcription factor, ABA-INDUCIBLE BHLH-TYPE TRANSCRIPTION FACTOR/JA-ASSOCIATED MYC2-LIKE1, acts as a repressor to negatively regulate jasmonate signaling in Arabidopsis. Plant Cell. 2013;25(5):1641-56.

33. Fujita Y, Fujita M, Shinozaki K, Yamaguchi-Shinozaki K. ABA-mediated transcriptional regulation in response to osmotic stress in plants. J Plant Res. 2011;124(4):509-25.
34. Wang Q, Wu C, Xie B, Liu Y, Cui J, Chen G, et al. Model analysing the antioxidant responses of leaves and roots of switchgrass to $\mathrm{NaCl}$-salinity stress. Plant Physiol Biochem. 2012;58:288-96.

35. Hu L, Li H, Pang H, Fu J. Responses of antioxidant gene, protein and enzymes to salinity stress in two genotypes of perennial ryegrass (Lolium perenne) differing in salt tolerance. J Plant Physiol. 2012;169(2):146-56.

36. Kim YS, Sakuraba Y, Han SH, Yoo SC, Paek NC. Mutation of the Arabidopsis NAC016 transcription factor delays leaf senescence. Plant Cell Physiol. 2013;54(10):1660-72.

37. Guo Y, Gan S. AtNAP, a NAC family transcription factor, has an important role in leaf senescence. Plant J. 2006;46(4):601-12.

38. Kim JH, Woo HR, Kim J, Lim PO, Lee IC, Choi SH, et al. Trifurcate feedforward regulation of age-dependent cell death involving miR164 in Arabidopsis. Science. 2009;323:1053-7.

39. Balazadeh S, Siddiqui H, Allu AD, Matallana-Ramirez LP, Caldana C, Mehrnia $M$, et al. A gene regulatory network controlled by the NAC transcription factor ANAC092/AtNAC2/ORE1 during salt-promoted senescence. Plant J. 2010;62(2):250-64

40. Sakuraba Y, Kim YS, Han SH, Lee BD, Paek NC. The Arabidopsis transcription factor NAC016 promotes drought stress responses by repressing AREB1 transcription through a trifurcate feed-forward regulatory loop involving NAP. Plant Cell. 2015;27(6):1771-87.

41. Mizoguchi M, Umezawa T, Nakashima K, Kidokoro S, Takasaki H, Fujita Y, et al. Two closely related subclass II SnRK2 protein kinases cooperatively regulate drought-inducible gene expression. Plant Cell Physiol. 2010;51(5):842-7.

42. Lu Y, Sasaki Y, Li X, Mori IC, Matsuura T, Hirayama T, et al. ABI1 regulates carbon/nitrogen-nutrient signal transduction independent of $A B A$ biosynthesis and canonical ABA signalling pathways in Arabidopsis. J Exp Bot. 2015;66(9):2763-71.

43. Osakabe Y, Arinaga N, Umezawa T, Katsura S, Nagamachi K, Tanaka H, et al. Osmotic stress responses and plant growth controlled by potassium transporters in Arabidopsis. Plant Cell. 2013;25(2):609-24.

44. Ha CV, Leyva-Gonzalez MA, Osakabe Y, Tran UT, Nishiyama R, Watanabe Y, et al. Positive regulatory role of strigolactone in plant responses to drought and salt stress. Proc Natl Acad Sci U S A. 2014;111(2):851-6.

45. Jensen MK, Lindemose S, de Masi F, Reimer JJ, Nielsen M, Perera V, et al. ATAF1 transcription factor directly regulates abscisic acid biosynthetic gene NCED3 in Arabidopsis thaliana. FEBS Open Bio. 2013;3:321-7.

46. Fujita M, Fujita Y, Maruyama K, Seki M, Hiratsu K, Ohme-Takagi M, et al. A dehydration-induced NAC protein, RD26, is involved in a novel ABAdependent stress-signaling pathway. Plant J. 2004;39(6):863-76.

47. Chen X, Wang Y, Lv B, Li J, Luo L, Lu S, et al. The NAC family transcription factor OSNAP confers abiotic stress response through the ABA pathway. Plant Cell Physiol. 2014;55(3):604-19.

48. Bu Q, Jiang H, Li CB, Zhai Q, Zhang J, Wu X, et al. Role of the Arabidopsis thaliana NAC transcription factors ANAC019 and ANAC055 in regulating jasmonic acid-signaled defense responses. Cell Res. 2008;18(7):756-67.

49. Park J, Kim YS, Kim SG, Jung JH, Woo JC, Park CM. Integration of auxin and salt signals by the NAC transcription factor NTM2 during seed germination in Arabidopsis. Plant Physiol. 2011;156(2):537-49.

50. Livak KJ, Schmittgen TD. Analysis of relative gene expression data using realtime quantitative PCR and the $2^{-\Delta \Delta C T}$ method. Methods. 2001;25(4):402-8.

51. Yoo SD, Cho YH, Sheen J. Arabidopsis mesophyll protoplasts: a versatile cell system for transient gene expression analysis. Nat Protoc. 2007;2(7):1565-72.

52. Clough SJ, Bent AF. Floral dip: a simplified method for Agrobacteriummediated transformation of Arabidopsis thaliana. Plant J. 1998;16(6):735-43.

53. Heath RL, Packer L. Photoperoxidation in isolated chloroplasts: I. Kinetics and stoichiometry of fatty acid peroxidation. Arch Biochem Biophys. 1968;125(1):189-98.

54. Arnon DI. Copper enzymes in isolated chloroplasts. Polyphenoloxidase in beta vulgaris. Plant Physiol. 1949;24(1):1-15.

55. Hu W, Yuan Q, Wang Y, Cai R, Deng X, Wang J, et al. Overexpression of a wheat aquaporin gene, $\mathrm{TaAQP} 8$, enhances salt stress tolerance in transgenic tobacco. Plant Cell Physiol. 2012;53(12):2127-41. 